\title{
Effects of an artificial pupil in visual perception
}

\author{
D. L. SCHURMAN, DEPARTMENT OF PSYCHOLOGY, \\ UNIVERSITY OF ILLINOIS, Urbana, Ill. 61801
}

The role of an artificial pupil in perception studies using backlighted stimuli is neither obvious nor has it been thoroughly explored. This study finds an effect which increases the signal/noise ratio of the stimulus. The artificial pupil increases the signal/noise ratio by increasing depth of field, increasing figure/ground contrast, and sharpening figure contours. The use of an artificial pupil is recommended in visual perception experiments.

The artificial pupil (i.e., an aperture between the light and the eye) is an integral component of the standard Maxwellian view apparatus. It has also been used in other visual apparatus for decades. Obviously, the artificial pupil limits the area of the retina illuminated and, thereby, the amount of illumination, when the presented stimulus is on a white field (i.e., frontlighted). However, when the stimulus is on a black field, or when it is a point source (i.e., backlighted), the function of the artificial pupil is not so obvious. In the backlighted case, the lighted portion of the stimulus field is smaller than the aperture, so that there seems to be no light limiting nor constant area function performed.

Nonetheless, the secondary literature indicates that an artificial pupil does perform some sort of function when used with backlighted stimuli. An artificial pupil appears necessary in investigations dealing with time/intensity reciprocity. Some investigators have expressed the opinion that the sharp break from $I x$ $T=K_{1}$ to $I=K_{2}$ cannot be found if an artificial pupil is not used in these experiments. 1

There appears to have been little or no systematic investigation of what the role of an artificial pupil might be when backlighted or when point source stimulation is used. Indeed, there has been no investigation of whether there is any effect at all. The study reported here attempts to make such a systematic investigation.

Procedure. The stimuli were the letters A, T, and U in News Gothic capital style, subtending $10 \mathrm{~min}$ of angle at the viewing distance of $38 \mathrm{in}$. The letters were white on black transparencies mounted in $35 \mathrm{~mm}$ slide holders. Each stimulus was presented an equal number of times in random order. Presentation was made in a one-channel tachistoscope at $40 \mathrm{msec}$ duration. The Ss viewed the stimuli monocularly with the dominant eye. A faintly glowing orange cross served as a fixation point. The $S$ initiated each trial by pressing a trigger when he had the fixation point sharply in focus.

A variable aperture artificial pupil, constructed from an iris diaphragm, was mounted in the visual alley 8 in. from S's eye. Four aperture sizes, $35 \mathrm{~mm}$, $20 \mathrm{~mm}, 15 \mathrm{~mm}$, and $10 \mathrm{~mm}$, were used. These apertures are equivalent to $4.38 \mathrm{~mm}, 2.50 \mathrm{~mm}, 1.88 \mathrm{~mm}$, and $1.25 \mathrm{~mm}$ at the eye.

Subjects were two female graduate students at the University of Illinois with normal or corrected to normal vision. They were given six blocks of 24 trials in each session for two and four practice sessions, respectively. In the last practice session, the luminance level required for approximately $75 \%$ recognition accuracy was determined using the $35 \mathrm{~mm}$ aperture. This level was $0.27 \mathrm{~mL}$ for one $\mathrm{S}$ and $0.13 \mathrm{~mL}$ for the other; these levels were used throughout the experiment. Two 24-trial blocks were run using each aperture size and were counterbalanced to control order effects.
Table 1

Per Cent Accuracy by Pupil Size

\begin{tabular}{lcccc} 
& \multicolumn{5}{c}{ Pupil diameter (mm) } \\
\cline { 2 - 5 } & 35 & 20 & 15 & 10 \\
\hline Per cent correct & 72.92 & 73.96 & 81.25 & 83.33 \\
\hline
\end{tabular}

Results. Since the results were very similar for the two Ss, the pooled per cent correct scores are given (Table 1). As can be seen, accuracy increases in an orderly manner as the diameter of the artificial pupil decreases. The difference between the pooled proportion correct for the $35 \mathrm{~mm}$ condition and the $10 \mathrm{~mm}$ condition was tested by a $t$ test between proportions. The $t$ value calculated was significant at the .01 level of confidence $(t=4.98$, $\mathrm{df}=70$ ).

Discussion. These Ss display a definite effect from an artificial pupil with backlighted stimuli. The explanation of this nonobvious effect can be determined from known facts of physical optics and eye physiology. An artificial aperture in front of the eye can be conceptualized as a lens of infinite focal length and zero curvature. It therefore eliminates stray and scattered light from point sources by a collimation effect. It also prevents light from entering the eye through the iris and/or sclera itself. The stimuli used in this study were of a line width of less than $1.5 \mathrm{~min}$ of angle and can be considered a line of point sources. That is, light scatter would occur with the forms used here as well as with a true point source. Limiting the angle at which light strikes the lens of the eye and the area of the lens in use, makes the eye lens functionally a more nearly perfect lens. This, in turn, reduces the amount of light scatter and aberration within the eye, increasing the actual figure/ground contrast on the retina and sharpening the contours of the figure.

In addition, the artificial pupil increases the depth of focus, just as the diaphragm of a camera increases its depth of field. Increasing the depth of field decreases the precision of focus required of $S$. Trial-to-trial judgments are therefore more consistent, and fatigue effects are minimized. The effects of accommodation drift (the change in S's focus between the time he decides to press the trigger and the time the stimulus appears) are also minimized. The exact relationship between depth of field and aperture size is dependent on the specifications of the lens in the system. These specifications are difficult to obtain in living Ss and so, quantification of this effect is not attempted here.

The two main functions of the artificial pupil, increasing depth of field and making the eye's lens functionally more ideal, have the effects of minimizing trial-to-trial variation, reducing fatigue and accommodation drift effects, and increasing the signal to noise ratio by increasing figure/ground contrast and sharpening contours. The overall effect is to reduce the amount of noise or "slop" in the data. Thus, the use of an artificial pupil when the entire Maxwellian view system is not feasible or undesirable has a real payoff in increasing the precision of the data. Since an artificial pupil is easy to install, its use would be well worth the trouble in most visual perception experiments.

NOTE

1. D. E. Kahneman, personal communication. 